\title{
Adnexal Torsion in Ovarian Hyperstimulation Syndrome in Early Pregnancy: A Diagnostic Challenge
}

\author{
Pradeep R Regmi ${ }^{1}$, Isha Amatya ${ }^{2}$, Atit Poudel ${ }^{3}$ \\ ${ }^{1}$ Department of Radiology, Faculty of Medicine, School of Health Sciences, University of Ioannina, Greece, ${ }^{2}$ Department \\ of Community Medicine, Kathmandu Medical College, Kathmandu, ${ }^{3}$ Department of Obstetrics and Gynecology,. \\ Hospital for Advanced Medicine and Surgery, Kathmandu
}

\section{Corresponding author:}

Pradeep R Regmi, MBBS, MD

Department of Radiology, Faculty of Medicine, School of Health Sciences, University of loannina, Greece

Email:pradeep.iom@gmail.com

Submitted : Feb 31, 2020

Accepted : Mar 30, 2020

\begin{abstract}
Adnexal torsion in ovarian hyperstimulation syndrome (OHSS) is rare but serious complication in pregnancy. It is more common in assisted reproductive techniques. Delay in diagnosis and treatment results in functional loss of ovary as well as termination of pregnancy. In this report, we are presenting a case of 27 years female with subfertility who conceived after ovulation induction presented at 7 weeks 6 days period of gestation with ovarian hyperstimulation associated with adnexal torsion. Later, she was treated with laparoscopic salpingo-oophorectomy.
\end{abstract}

Keywords: Adnexal torsion, ovarian hyperstimulation syndrome, pregnancy

\section{INTRODUCTION}

W

idespread use of assisted reproductive techniques (ART) has increased the complications like ovarian hyperstimulation syndrome (OHSS), ovarian torsion, ectopic pregnancy and heterotropic pregnancy. OHSS is a serious iatrogenic complication due to the drugs used for ovarian stimulation. Its incidence in clomiphene induction is about $13.5 \%$ and $8 \%$ in mild and moderate form. ${ }^{1}$ Severe form is quiet rare with clomiphene induction. There are few case reports of ovarian torsion in OHSS in early pregnancy. ${ }^{2,3}$ Here we describe a case conceived after ovarian stimulation and presented at $7^{\text {th }}$ week of gestation with features of OHSS and adnexal torsion.

\section{CASE PRESENTATION}

A 24 years female with primary subfertility conceived after ovulation induction with letrozole.
She presented to Emergency Department of Hospital for Advanced medicine and Surgery (HAMS) at $7^{+6}$ weeks of gestation with severe right sided abdominal pain, nausea, and vomiting for 7-8 hrs. Pain abdomen was sudden in onset, moderate to severe in intensity, on and off, dull to sharp aching, with no radiation and no aggravating and relieving factors. It was associated with vomiting 6-7 episodes, non-projectile, containing food particles, non-bilious and non-blood mixed. There were no history of PN bleeding or discharge, fever, headache, shortness of breath, cough, blurring of vision or loss of consciousness. Bowel and bladder habit were normal. No significant personal, surgical and family history. On clinical examination, vital signs were stable. Abdomen was soft but tender and mass was felt up to umbilical level. On per speculum examination, cervix appeared healthy. Uterus was corresponding to 8 weeks size and large mass was felt on right adnexa on PN examination.

USG showed huge hypoechoic ovary about 400 

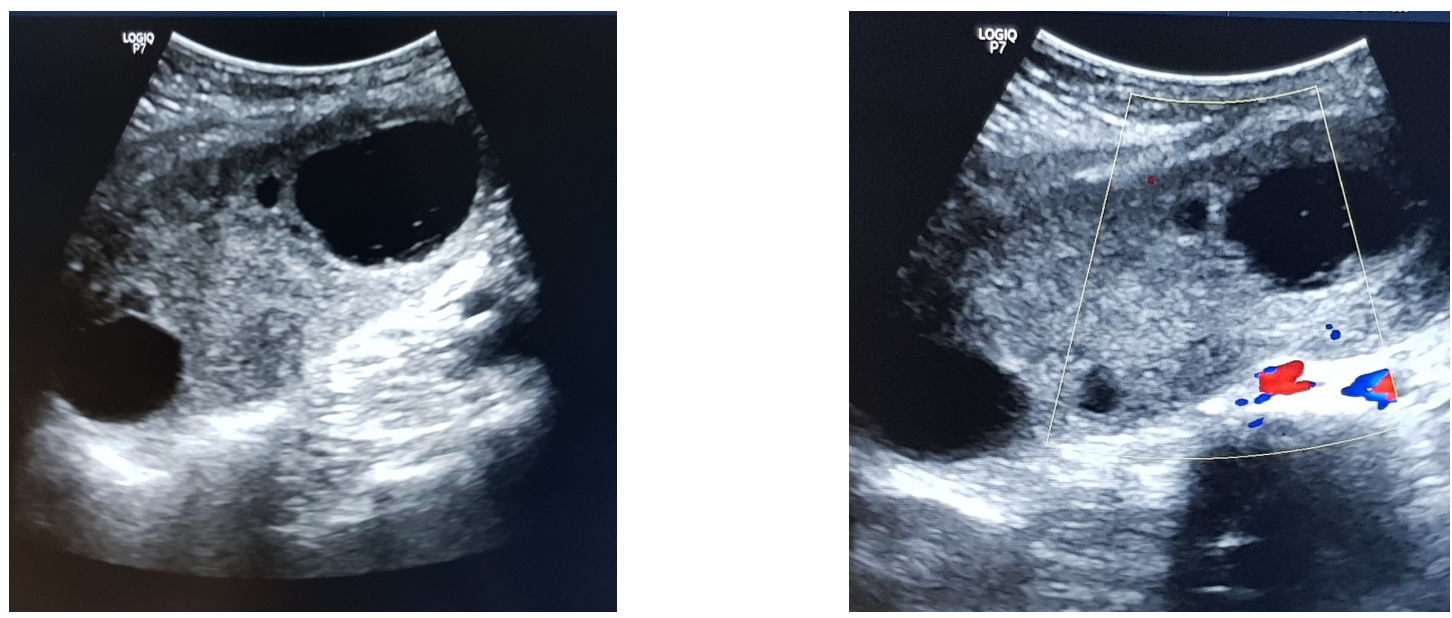

Fig 1a. Enlarged hypoechoic right ovary showing cysts in peripheral location. Fig $1 b$. No vascularity is seen within the enlarged ovary on color Doppler study

cc with few cystic areas and largest cyst of 5.5 $\mathrm{cm}$ (Figure 1a). There was no vascularity seen in color Doppler study suggestive of high possibility of adnexal torsion (Figure 1b). A single intrauterine viable pregnancy of 7 weeks period of gestation was seen (Figure 2a). Minimal free fluid was seen in pelvic cavity. Left ovary appear normal in size. There were no free fluid in bilateral pleural and pericardial cavity. Then, emergency diagnostic laproscopic surgery was done which revealed greyish-black huge right ovary with right adnexal torsion (Figure 2b). Therefore, right sapingo-oophorectomy was performed. Post-operative period was uneventful and discharged. Pregnancy was continued normally.

\section{DISCUSSION}

Ovarian torsion is rare in pregnancy. Enlarged hyperstimulated ovaries are at risk of torsion and is reported as 11 times more common in assisted reproductive technique (ART) than nonART pregnancies. ${ }^{1}$ The possible pathogenesis is the large cysts within the ovary serve as the lead point for twisting of the vascular pedicle along with pelvic ascites. ${ }^{1,2}$ Ovarian torsion should be suspected and ruled out whenever the female patient undergoing ovarian stimulation or in vitrofertilisation (IVF) presented with severe abdominal pain. Although superovulation can increase the chance of pregnancy by increasing the number of oocytes, it also raises the possibility of developing ovarian hyper stimulation syndrome (OHSS). OHSS is a broad spectrum of signs and symptoms that include abdominal distention and discomfort, enlarged ovary, ascites and other complications of enhanced vascular permeability. ${ }^{4}$ Ultrasound is the primary modality. The characteristic findings of "wheel spoke appearance" is seen in OHSS with the peripheral location of follicles and central stroma. The scenario becomes more challenging when
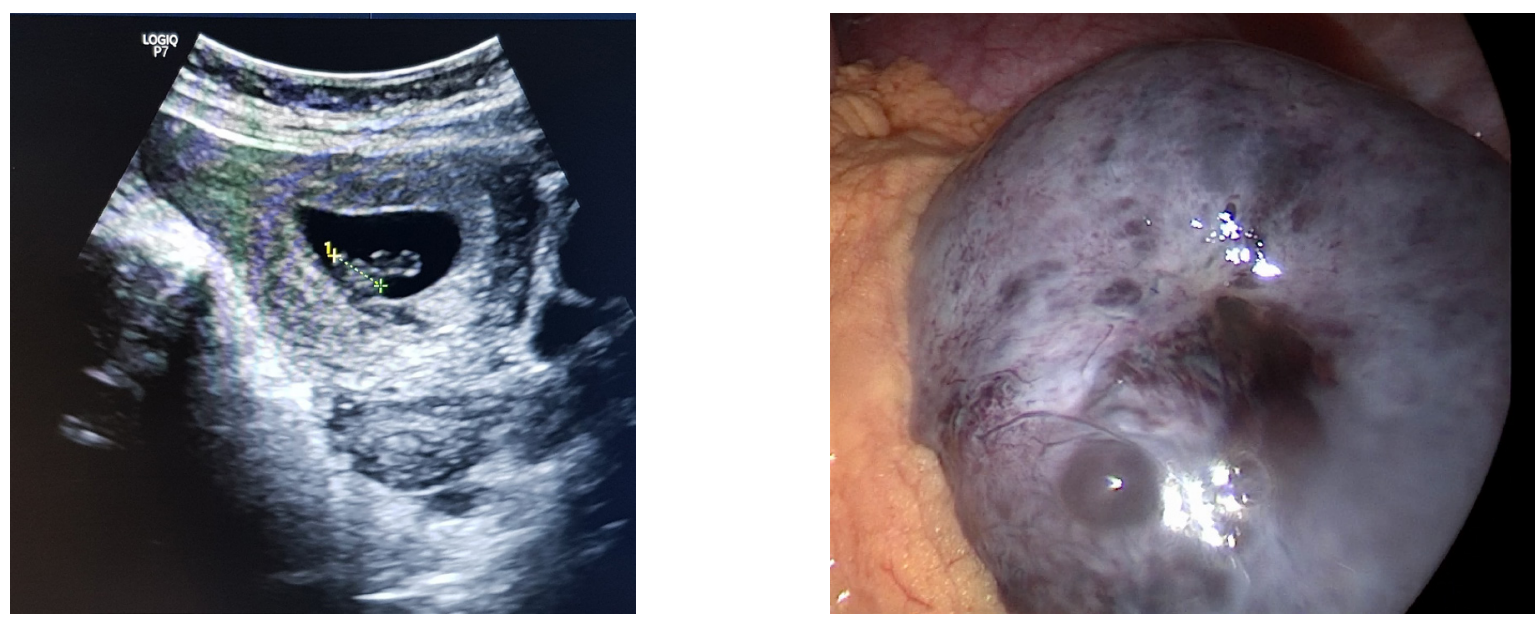

Fig 2a. Single intrauterine gestational sac of 7 weeks 6 days period of gestation. Arrow shows the measurement of Crown-rump Length. Fig 2b. Greyish black discolored right ovary during laproscopic surgery 
is associated with torsion. The most consistent finding of torsion in ultrasound is asymmetrically enlarged unilateral ovary usually the right. Pelvic ascites is present up to $87 \%$ of the cases. ${ }^{1}$ Other associated findings are twisted vascular pedicle, string of pearl signs (peripherally located multiple small less than $25 \mathrm{~mm}$ in enlarged ovary). It is associated with heterogenous central stroma due to edema and haemorrhage and peripherally displaced follicles. However, twisted vascular pedicle is not clearly visible in our case. Absence of vascularity in colour Doppler is specific but not sensitive to diagnose ovarian torsion. Only $73 \%$ of the cases confirmed with ovarian torsion noted the absence of arterial flow in colour Doppler study., Due to these aforementioned features of enlarged ovary with peripherally displaced follicles which are in common with both ovarian hyperstimulation syndrome and ovarian torsion, color Doppler evaluation and adequate correlation with clinical status of the patients is crucial in diagnosis of torsion in hyperstimulated ovary and guide the next step for management. Diagnostic laproscopic evaluation in equivocal cases are indicated followed by derotation of the involved adnexa in the same setting if the involved ovary appears viable. Otherwise, adnexectomy is indicated in the cases of non-viable ovaries. ${ }^{3}$

\section{CONCLUSION}

Considering the increase in number of ovulation induction, OHSS and its associated complications including ovarian torsion is likely to be more common than in the past. Early diagnosis and appropriate surgical management of ovarian torsion is required for the continuation of the pregnancy. Laparoscopic surgery in early pregnancy is safe and useful strategy for equivocal cases. Radiologists and gynecologists should be working together and correlate the findings for the early diagnosis and thereby appropriate surgical management.

\section{CONFLICT OF INTEREST}

None declared.

\section{REFERENCES}

1. Baron KT, Babagbemi KT, Arleo EK, Asrani AV, Troiano RN. Emergent complications of assisted reproduction: expecting the unexpected. Radiographics. 2013 Jan;33(1):229-44.

2. Navarro NG, Grau EG, Pérez SP, Luna LR. Ovarian torsion and spontaneous ovarian hyperstimulation syndrome in a twin pregnancy: a case report. International journal of surgery case reports. 2017 Jan 1;34:66-8.

3. Spitzer D, Wirleitner B, Steiner H, Zech N. Adnexal Torsion in Pregnancy after Assisted Reproduction - Case Study and Review of the Literature. Geburtsh Frauenheilk. 2012 Aug;72(08):716-20.

4. Cabar FR. Ovarian hyperstimulation syndrome in a spontaneous singleton pregnancy. Einstein (São Paulo). 2016 Jun;14(2):231-4.

5. Chang HC, Bhatt S, Dogra VS. Pearls and pitfalls in diagnosis of ovarian torsion. Radiographics. 2008 Sep;28(5):1355-68. 